\title{
Cloning of a novel levoglucosan kinase gene from Lipomyces starkeyi and its expression in Escherichia coli
}

\author{
Jianghong Dai $\cdot$ Zhisheng Yu $\cdot$ Yongzhi He \\ Ling Zhang $\cdot$ Zhihui Bai $\cdot$ Zhiyang Dong $\cdot$ \\ Yuguo Du $\cdot$ Hongxun Zhang
}

Received: 1 March 2009/Accepted: 16 April 2009/Published online: 29 April 2009

(C) Springer Science+Business Media B.V. 2009

\begin{abstract}
Levoglucosan, cellulosic pyrolysate, is converted to glucose-6-phosphate by a specific levoglucosan kinase in fungi. A novel cDNA of levoglucosan kinase gene (lgk) from yeast Lipomyces starkeyi YZ-215 was isolated by RACE method. The 1,445 bp cDNA fragment $(l g k)$ harbouring the kinase gene exhibited one open reading frame (ORF) composed of 1,317 bp flanked by a $14 \mathrm{bp}$ 5'-UTR and a 114 bp 3'-UTR, including a 25 bp poly(A) tail. The ORF encoded a 439 amino acid polypeptide with a $48.4 \mathrm{kDa}$ predicted molecular mass. Analysis of amino sequence revealed that the kinase belonged to the bacterial anhydro- $N$-acetylmuramic acid kinase (AnmK) family, and kinase-like proteins existed in some fungi, especially in filamentous fungi such as Aspergillus. The kinase gene was transformed into Escherichia coli BL21 (DE3), recombinant $E$. coli could grow in M9 minimal medium with levoglucosan as a sole carbon source when induced by IPTG. In addition, the recombinant kinase was overexpressed, purified and characterized. The kinase was stable at $\mathrm{pH} 7-10$ and showed maximum activity at $30^{\circ} \mathrm{C}$ and $\mathrm{pH}$ 9.0 as natural kinase, but presented higher thermostability. Kinetic constants (apparent $K_{\mathrm{m}}$ values) for LG and ATP
\end{abstract}

J. Dai $\cdot$ Z. Yu $(\bowtie) \cdot$ H. Zhang

College of Resources \& Environment, Graduate University

of the Chinese Academy of Sciences, 100049 Beijing,

People's Republic of China

e-mail: yuzs@gucas.ac.cn

Y. He $\cdot$ L. Zhang $\cdot$ Z. Dong

Institute of Microbiology, Chinese Academy of Sciences,

100101 Beijing, People's Republic of China

Z. Bai $\cdot$ Y. Du $\cdot$ H. Zhang

Research Center for Eco-Environmental Sciences,

Chinese Academy of Sciences, 100085 Beijing,

People's Republic of China were $105.3 \pm 12.5$ and $0.20 \pm 0.02 \mathrm{mM}$, respectively. Furthermore, the kinase showed substrate specificity for LG. This novel levoglucosan kinase gene would be useful in constructing recombinant microbial strains for the efficient bioconversion of cellulosic pyrolysate to ethanol.

Keywords Levoglucosan kinase gene .

Lipomyces starkeyi YZ-215 - RACE · Cellulose pyrolysis

\section{Introduction}

Levoglucosan (1,6-anhydro- $\beta$-D-glucopyranose, LG) is very scarce in nature compared with other natural carbohydrates such as glucose, sucrose and fructose, and mainly exists in derivatives from natural pyrolysis of cellulosic materials such as woody and herbaceous biomass (Gao et al. 2003; Schauer et al. 2001). However, LG can be obtained in bulk under appropriate pyrolysis conditions using cellulosic materials (Brown et al. 2001; Prosen et al. 1993). LG can be used as a potential fermentation carbon and energy source in the fermentation industry, because LG can be metabolized directly by microorganisms or indirectly through mild acid hydrolysis to produce glucose (Nakagawa et al. 1984; Yu and Zhang 2003). Nakagawa et al. (1984) and Zhuang et al. (2001) have reported its use in itaconic acid and citric acid fermentation by Aspergillus terreus and Aspergillus niger, respectively.

As for direct bioconversion of LG, eukaryotic microorganisms have a different metabolic pathway from prokaryotic organisms. In Arthrobacter bacteria, LG is transformed into glucose through at least three enzymatic reactions (Nakahara et al. 1994). In yeasts and filamentous fungi, LG is directly converted to glucose-6-phosphate (one-step) by a specific levoglucosan kinase (LGK) 
(Kitamura et al. 1991; Zhuang and Zhang 2002). As shown in Fig. 1, the 1,6-anhydro bond of LG is cleaved and simultaneously phosphorylated by LGK in the presence of $\mathrm{Mg}^{2+}$ and ATP. Apparently, one-step direct bioconversion of LG in fungi is more appealing.

In recent years, as an alternative energy product, cellulosic ethanol has been widely expected (Farrell et al. 2006; Waltz 2008). However, ethanol could not be efficiently produced by fermentation of LG with microorganisms. Prosen et al. (1993) found five yeast strains that could ferment activated charcoal-treated pyrolysis tar with high concentration of LG to ethanol. However, the ethanol yields were considerably low, compared with glucose as the fermentation carbon source. Up to now, about 43 species distributed in 19 genera of fungi have been screened with LG-assimilating capability (Kitamura et al. 1991; Kitamura and Yasui 1991; Nakagawa et al. 1984; Prosen et al. 1993; Xie et al. 2006; Yu 2003). Unfortunately, few strains can produce ethanol, or the conversion ratio is low. Furthermore, all identified LGKs have low affinity for LG with high $K_{\mathrm{m}}$ values from 48 to $102 \mathrm{mM}$ (Kitamura and Yasui 1991; Xie et al. 2006), and it is speculated that LGK would be an alternatively functioning protein which incidentally had an affinity for LG. This awfully embarrasses direct bioconversion of LG. Therefore, exploitation of efficient recombinant microbial strains to ferment $\mathrm{LG}$ to produce ethanol is an alternative.

At present, the reports on LGK and its gene $(\lg k)$ remain very limited. Zhang and Zhang (2002) constructed a cDNA library of Aspergillus niger, three positive clones were obtained. However, the specific activity of recombinant LGK was only approximately one third of natural LGK. Consequently, Xie et al. (2005) purified LGK from A. niger and identified a 14-mer peptide by LC-ESI-MS/MS. The cDNA of lgk gene was then isolated using RT-PCR and one degenerated primer, two positive clones were obtained. However, the deduced amino sequence showed no similarity to that of any protein kinase, and recombinant LGK had low activity (0.05 U/mg). As a result, a LGK, with relatively high activity for LG from yeast $L$. starkeyi YZ-215, was recently purified and characterized, and three peptides (TGLTSLTDFR, GKVDQELVDDFLK,

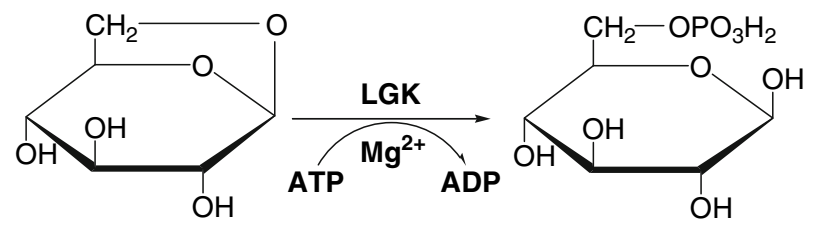

Levoglucosan

Glucose-6-phosphate

Fig. 1 Catalytic mechanism of LGK in fungi
LGSPDDLVATTTR) were sequenced by ESI-Q-TOF MS/ MS (Ning et al. 2008).

In this study, we isolated the cDNA of $l g k$ gene from L. starkeyi YZ-215 based on the above identified peptides, successfully expressed it in E. coli, and partially characterized recombinant LGK. To our knowledge, the lgk gene isolated in this study is a novel gene responsible for the decomposition of LG in fungi, and it would be useful material in construction of engineering strains for fermenting cellulosic pyrolysate to ethanol in the future.

\section{Materials and methods}

Strains, plasmids and media

L. starkeyi YZ-215 (Yu 2003) was employed as a lgk gene donor through this study. YPD medium (Adams et al. 2001) was used for cultivation of L. starkeyi. LG liquid medium (g/l: LG 10, yeast extract 2, $\mathrm{NaNO}_{3} 5, \mathrm{KH}_{2} \mathrm{PO}_{4} 1$, $\mathrm{MgSO}_{4} \cdot 7 \mathrm{H}_{2} \mathrm{O}$ 0.5, $\mathrm{FeSO}_{4}$ 0.01) and LGK induction medium (g/l: LG 10, $\mathrm{KH}_{2} \mathrm{PO}_{4} 1, \mathrm{MgSO}_{4} \cdot 7 \mathrm{H}_{2} \mathrm{O}$ 0.5) were used for isolation of total RNA. E. coli TOPO10 (Invitrogen, Carlsbad, CA) was used as the host for cloning vector pCR $4^{\circledR}-$ TOPO $^{\circledR}$ (Invitrogen). E. coli BL21 (DE3) (Novagen) was used as the host for expression vector pET21a(+) (Novagen). M9 minimal medium (Sambrook and Russell 2001) and chemically defined (CD) minimal medium (g/l: glucose 1, $\left(\mathrm{NH}_{4}\right)_{2} \mathrm{SO}_{4} \quad 5, \mathrm{KH}_{2} \mathrm{PO}_{4} \quad$, $\mathrm{MgSO}_{4} \cdot 7 \mathrm{H}_{2} \mathrm{O}$ 0.5, $\mathrm{pH} 5.0$ ) were used to test strains' ability to utilize different substrates.

\section{Isolation of total RNA and RT-PCR amplification of $l g k$} gene

A fresh clone of L. starkeyi in YPD plate was inoculated into $\mathrm{LG}$ liquid medium and incubated at $30^{\circ} \mathrm{C}$. The midlogarithmic culture was then centrifuged and transferred to LGK induction medium and cultivated for another $5 \mathrm{~h}$. The culture was collected and ground in liquid nitrogen, and then total RNA was extracted with TRNzol total RNA reagent following the manufacturer's (Tiangen) protocols.

The 'rapid amplification of cDNA ends' (RACE) method was applied for the amplification of $l g k$ gene. The synthesis of first-strand cDNA and RACE amplification were carried out with GeneRacer ${ }^{\mathrm{TM}}$ Kit (Invitrogen). Four degenerated gene-specific primers were determined according to two identified peptides GKVDQELVDDFLK and LGSPDDLVATTTR (Ning et al. 2008); P1: 5' ARGTIGAYCARGARYTIGT $3^{\prime}$ (sense, corresponding to the amino sequence KVDQELV), P2: 5' YTCYTGRTCIACY TTICC $3^{\prime}$ (antisense, corresponding to the amino sequence GKVDQE); P3: 5' GAYGAYYTIGTIGCIACIAC $3^{\prime}$ 
(sense, corresponding to the amino sequence DDLVATT), P4: 5' IGTIGCIACIARRTCRTC $3^{\prime}$ (antisense, corresponding to the amino sequence DDLVAT). For $3^{\prime}$ RACE amplification, first PCR was carried out with P1 and GeneRacer $^{\mathrm{TM}} 3^{\prime}$ Primer, then Nested PCR with P3 and GeneRacer $^{\mathrm{TM}} 3^{\prime}$ Nested Primer; for $5^{\prime}$ RACE amplification, first PCR was carried out with P4 and GeneRacer ${ }^{\mathrm{TM}} 5^{\prime}$ Primer, then Nested PCR with P2 and GeneRacer ${ }^{\mathrm{TM}} 5^{\prime}$ Nested Primer. The PCR products were ligated into pCR $4{ }^{\circledR}-\mathrm{TOPO}^{\circledR}$ vector and sequenced. The primers of $3^{\prime}$ end and $5^{\prime}$ end of $l g k$ gene were then synthesized, and the full-length cDNA of $l g k$ gene was ultimately acquired.

\section{Expression and purification of recombinant LGK}

The coding region of $l g k$ gene was ligated into pET-21a vector, and the resulted plasmid $\mathrm{pET}$-lgk, carrying $l g k$ gene under the control of the $\mathrm{T} 7$ promoter, was transformed into E. coli BL21 (DE3). Cells carrying pET-lgk were grown at $37^{\circ} \mathrm{C}$ with vigorous shaking in $100 \mathrm{ml}$ liquid LB medium containing $100 \mu \mathrm{g}$ of ampicillin/ml. After the culture grew to the mid-log phase, isopropyl- $\beta$-D-thiogalactopyranoside (IPTG) was added to a final concentration of $0.1 \mathrm{mM}$, and incubation was continued at $30^{\circ} \mathrm{C}$ for another $3 \mathrm{~h}$. Aliquot culture about $1 \mathrm{ml}$ was pelleted by centrifugation and total cell proteins were analyzed by SDS-PAGE. Remainder of culture was harvested by centrifugation and washed twice with $20 \mathrm{mM}$ Tris- $\mathrm{HCl}$ buffer $(\mathrm{pH} 8.0)$ containing $1 \mathrm{mM}$ ethylene diamine tetraacetic acid (EDTA), $1 \mathrm{mM}$ dithiothreitol (DTT) and $0.1 \mathrm{mM}$ phenylmethylsulfonyl fluoride (PMSF). Cells were then suspended in $20 \mathrm{ml}$ buffer and ruptured by sonication. After centrifugation at $20,000 \mathrm{~g}$ for $20 \mathrm{~min}$ at $4^{\circ} \mathrm{C}$, the supernatants were collected for purification.

Purification works were carried out following the procedures as described by Ning et al. (2008). All enzyme purification procedures were performed at $4^{\circ} \mathrm{C}$. In brief, the crude extract was treated by $40 \%$ ammonium sulfate and the supernatant was precipitated by $65 \%$ ammonium sulfate. The pellet was dissolved in $20 \mathrm{mM}$ Tris-HCl buffer ( $\mathrm{pH} 8.0$, $1 \mathrm{mM}$ EDTA, $1 \mathrm{mM}$ DTT) and pretreated using an Amicon Ultra-15, $10 \mathrm{~K}$ centrifugal filter device (Millipore), followed by sequential chromatographic steps with HiTrap Desalting column, Resource Q and Superdex 200 ${ }^{\mathrm{TM}} 200$ 10/300 GL columns (Amersham Biosciences, USA). During the chromatographic steps, fractions with high kinase activity were pooled, and the manipulations were performed on an Akta FPLC system (Amersham-Pharmacia Biotech).

\section{Enzyme assays}

Enzyme assays were carried out following the procedures as described by Ning et al. (2008). The reaction buffer was
$50 \mathrm{mM}$ Tris- $\mathrm{HCl}$ buffer ( $\mathrm{pH} 9.0$ ), and the reaction mixture contained $75 \mathrm{mM}$ LG, $2 \mathrm{mM}$ ATP, $0.2 \mathrm{mM}$ NADP, $10 \mathrm{mM} \mathrm{MgCl}_{2}$ and $1 \mathrm{U}$ glucose-6-phosphate dehydrogenase. Total soluble protein concentration was determined by the Bradford method (Tiangen) using bovine serum albumin (BSA) as the standard.

\section{Characterization of recombinant LGK}

The effects of temperature between 15 and $45^{\circ} \mathrm{C}$ on enzyme activity and stability were determined. Thermostability was determined by incubating the enzyme for $30 \mathrm{~min}$ at the designated temperature in $50 \mathrm{mM}$ Tris- $\mathrm{HCl}$ buffer ( $\mathrm{pH} 9.0$ ). The effects of $\mathrm{pH}$ on enzyme activity were determined by varying the substrate $\mathrm{pH}$ between 5.5 and 11.0. The buffers used were $50 \mathrm{mM}$ sodium citrate for $\mathrm{pH}$ 5.5-6.5, $50 \mathrm{mM}$ triethanolamine- $\mathrm{HCl} / \mathrm{NaOH}$ for $\mathrm{pH} 7.0$ 9.0, and $50 \mathrm{mM}$ glycine- $\mathrm{NaOH}$ for $\mathrm{pH} 9.0-11.0$. To determine the $\mathrm{pH}$ stability of enzyme, the enzyme was incubated at the designated $\mathrm{pH}$ for $30 \mathrm{~min}$ at $30^{\circ} \mathrm{C}$ and the residual activity was then measured in $50 \mathrm{mM}$ Tris- $\mathrm{HCl}$ buffer ( $\mathrm{pH}$ 9.0). The apparent $K_{\mathrm{m}}$ values were measured by determining the enzymatic activity at substrate concentration ranging from 10 to $100 \mathrm{mM}$ for $\mathrm{LG}$ and 0.2 to $2 \mathrm{mM}$ for ATP. To determine the substrate specificity, the reaction mixture contained LG and its different analogs, and concentration was $25 \mathrm{mM}$, respectively.

\section{Chemicals}

Analogs of LG: 1,6 -anhydro- $\beta$-D-glucosamine (anhGlcN) and 1,6-anhydro- $N$-acetyl- $\beta$-D-glucosamine (anhGlcNAc), both with $98 \%$ purity, were synthesized in Beijing Carbomex Biotech Co., Ltd.

\section{Results and discussion}

Cloning and characterization of $l g k$ gene from L. starkeyi YZ-215

In order to isolate $\lg k$ gene, RT-PCR amplification was applied to clone the gene. Four degenerate primers were designed from two of three identified peptides (Ning et al. 2008), and 5' RACE and $3^{\prime}$ RACE amplifications were conducted. Two fragments about 761 and 555 bp corresponding to the $5^{\prime}$ and $3^{\prime}$ region were obtained, respectively. The deduced amino sequence of $5^{\prime}$ fragment contained the third identified peptide TGLTSLTDFR. The full-length cDNA was a $1,445 \mathrm{bp}$ fragment containing a 1,317 bp open reading frame (ORF), which was flanked by a 14 bp 5'-UTR and a 114 bp 3'-UTR, including a 25 bp poly(A) tail. The stop codon was TAATGA with two 
Fig. 2 Phylogenetic analysis of LGK from L. starkeyi YZ-215, LGK-like proteins from fungi and AnmK from E. coli MG1655. The phylogenetic relationship was analyzed by the ClutalX2.03 (Thompson et al. 1997) program and drawn by TreeView 1.6.6 (Roderic, D. M. Page, http://taxonomy.zoology. gla.ac.uk/rod/rod.html) program. Numbers show results from bootstrap analyses $(1,000$ bootstrap replicates). Protein accession numbers: [1], EDU45785; [2], EDN99772; [3], EDN25583; [4], EAQ93900; [5], EAW25579; [6], EAL84290; [7], EDP47186; [8], EAA29125; [9], EAW24529; [10], EDP50956; [11], EAL86194; [12], EDK40502; [13], ABN66269; [14], EAW22768; [15], EAQ84482; [16], EAA32985; [17], CAK44911; [18], EAU33314; [19], BAE65712; [20], EED45075; [21], EAW24693; [22], EDP50844; [23], EAL89335; [24], EAA59060; [25], CAP80499; [26], EED14977; LGK: ACE79748; AnmK, AAC74712

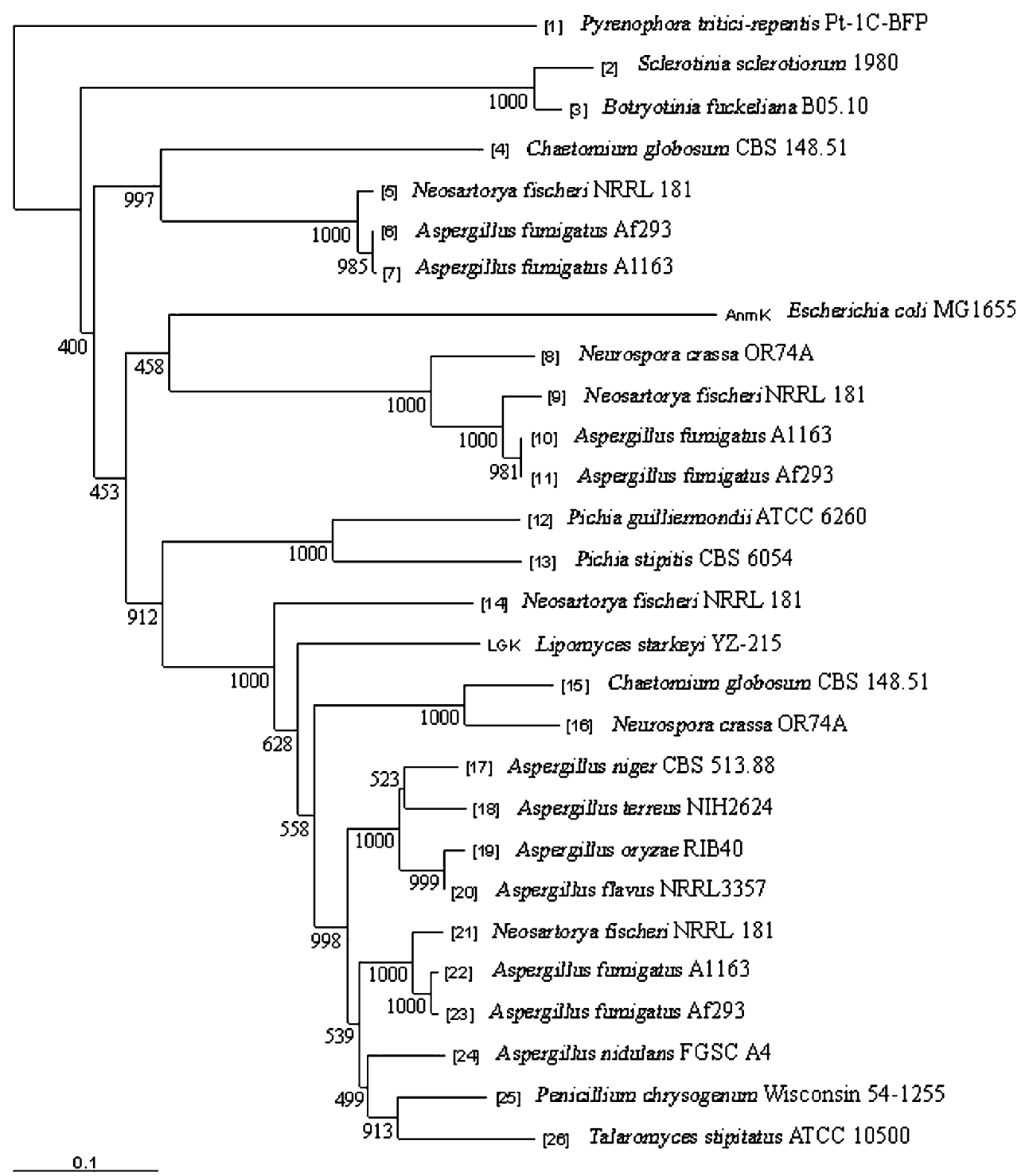

continuous stop codons and was followed by several other stop codons that were both in and out of frame. The ORF encoded a 439 amino acid polypeptide with a $48.4 \mathrm{kDa}$ predicted molecular mass, which is similar to natural LGK's mass, 48 and $47.7 \mathrm{kDa}$, identified by SDS-PAGE and MALDI-TOF MS (Ning et al. 2008), respectively. This suggests that LGK would not be glycosylated in L. starkeyi. No signal peptide was found with SignalP 3.0 program (http://www.cbs.dtu.dk/services/SignalP/). The nucleotide sequence of $l g k$ gene was deposited in the GenBank database under accession number EU751287.

Comparison and alignment of amino acid sequences

The deduced amino sequence of $l g k$ gene was searched in NCBI (http://www.ncbi.nlm.nih.gov/) using the BLASTP program. The results indicated that LGK belonged to the bacterial anhydro- $N$-acetylmuramic acid kinase (AnmK) family which is Clusters of Orthologous Groups (COGs). The AnmK family was sorted into two groups: one group was AnmK which existed widely in bacteria, and the other was LGK-like proteins which existed in some fungi, especially in filamentous fungi such as Aspergillus. Some fungi have multiple LGK-like proteins. For example, Aspergillus fumigatus has three variant multiples, Neosartorya fischeri has four. In addition, A. niger has LGK-like protein, yet it showed no similarity to the LGK reported in A. niger (Xie et al. 2005). In particular, it is worth noting that all sequenced yeast strains of Saccharomyces cerevisiae do not have LGK-like protein. This accounts for why only one strain of $S$. cerevisiae with LG-assimilating capability has been screened up to now (Prosen et al. 1993).

In the AnmK family, the function of AnmK was approved by experiments in E. coli (Uehara et al. 2005), whereas LGK-like proteins in fungi were hypothetical 
proteins. Similar to the catalytic mechanism of LGK, AnmK cleaves the 1,6-anhydro bond of 1,6-anhydro- $N$ acetylmuramic acid (anhMurNAc) and simultaneously phosphorylates the sugar to form $N$-acetylmuramic acid-6phosphate (MurNAc-P). LGK shares $33.0 \%$ amino acid identity to AnmK.

Multiple alignments of amino acid sequence were performed using the ClustalX2.03 program (Thompson et al. 1997). The results revealed that LGK had a variant identity from 29.9 to $77.5 \%$ to LGK-like proteins in fungi with the average of $52.3 \%$, while AnmK had a variant identity from 20.4 to $37.1 \%$ to LGK-like proteins with the average of $27.1 \%$. A phylogenetic tree of LGK, LGK-like proteins and AnmK based on multiple alignments is shown in Fig. 2.

At present, owing to limited fungi genomes decoded, only 16 species were presented LGK-like proteins. Considering the $\mathrm{N}$-acetylmuramic acid (NAM) not existing in fungi, it is suggested that LGK-like proteins would be levoglucosan kinases in fungi.

\section{Expression of $l g k$ gene in E. coli}

Expression plasimid pET-lgk, together with empty pET21a as a control, was transformed into $E$. coli. The maximum activity of the crude extract was $7.9 \mathrm{U} / \mathrm{mg}$. The analysis of cell free extract by SDS-PAGE shows that

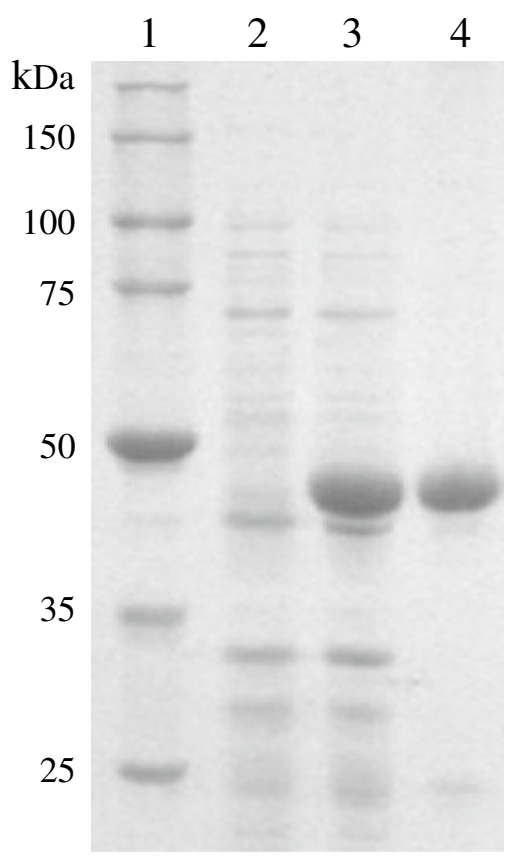

Fig. 3 SDS-PAGE profile of recombinant LGK overexpressed in E. coli BL21 (DE3)/pET-lgk. The protein samples were separated on $12.0 \%$ SDS-PAGE with Coomassie blue R-250. Lane 1 protein molecular weight marker; lane 2 crude extract of E. coli BL21 (DE3)/ pET-21a; lane 3 crude extract of $E$. coli BL21 (DE3)/pET-lgk; lane 4 purified recombinant LGK

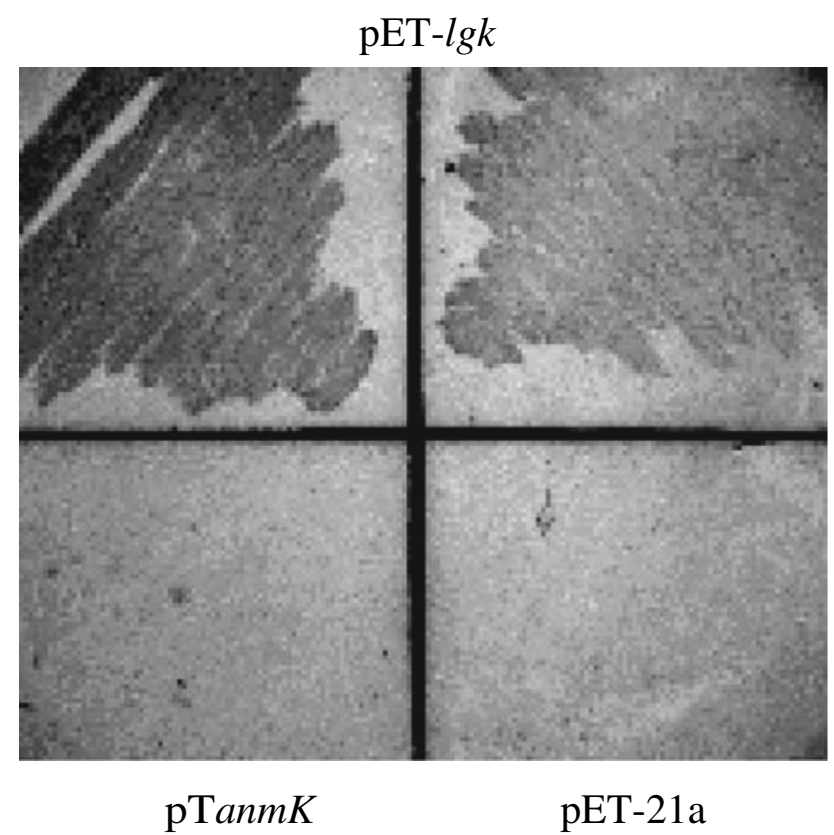

Fig. 4 Growth of E. coli BL21 (DE3)/pET-lgk in M9 minimal medium plate. The top panel is E. coli BL21 (DE3)/pET-lgk, the lower panel are controls E. coli BL21 (DE3)/pTanmK and E. coli BL21 (DE3)/pET-21a. M9 minimal medium was modified with $1.0 \%$ LG instead of glucose; concentration of IPTG was $0.1 \mathrm{mM}$. E. coli BL21 (DE3)/pTanmK (Uehara et al. 2005), a kind gift from Professor Dr. James T. Park, carries anmK gene under the control of the T7 promoter of pGEM-T
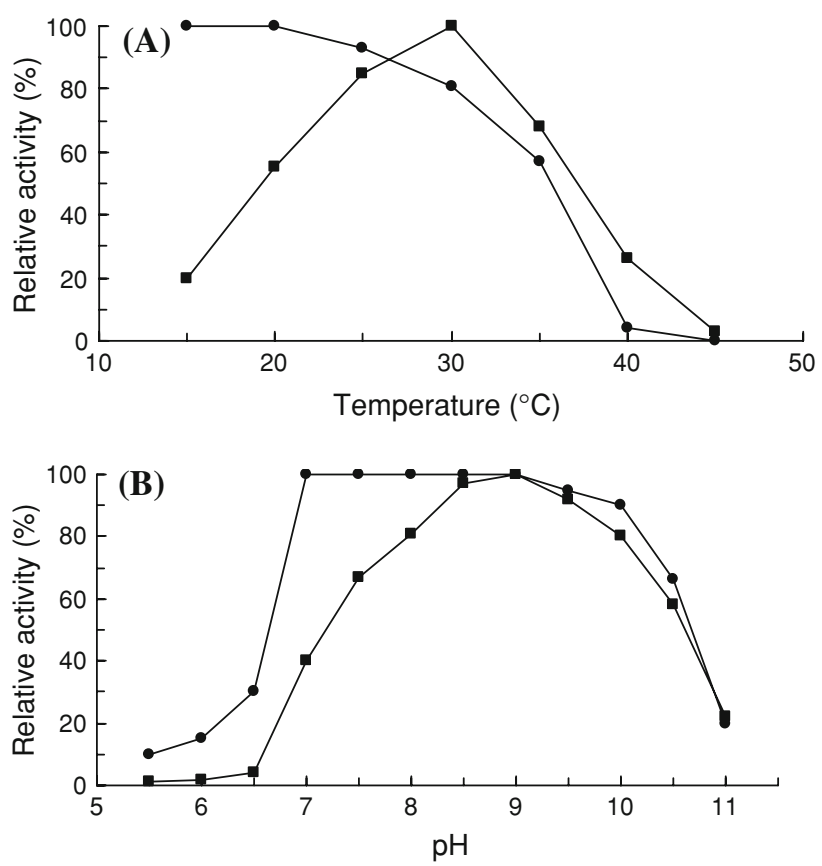

Fig. 5 Effects of temperature (A) and $\mathrm{pH}(\mathbf{B})$ on the activity ( $(\mathbf{\square})$ and stability () of recombinant LGK from E. coli BL21 (DE3)/pET-lgk 
Table 1 Growth comparison of pET-21a, pTanmK, pET-lgk and L. starkeyi in liquid MM

\begin{tabular}{|c|c|c|c|c|c|}
\hline \multirow[t]{2}{*}{ Strains } & \multicolumn{5}{|c|}{ Carbon sources in $\mathrm{MM}$} \\
\hline & LG & anhGlcN & anhGlcNAc & GlcN & GlcNAc \\
\hline pET-21a & - & - & - & +++++ & +++++ \\
\hline pTanmK $K^{\mathrm{a}}$ & - & - & + & +++++ & +++++ \\
\hline pET-lgk $k^{\mathrm{a}}$ & +++++ & +++ & ++ & +++++ & +++++ \\
\hline L. starkeyi ${ }^{\mathrm{b}}$ & +++++ & - & - & - & - \\
\hline
\end{tabular}

" +++++ " denotes stationary phase of growth. Concentration of carbon source was $1.0 \%$, respectively. Temperature of cultivation was $30^{\circ} \mathrm{C}$, time was about $72 \mathrm{~h}$. The tests were conducted in small vials containing $5 \mathrm{ml}$ liquid MM

${ }^{a}$ M9 minimal medium

b CD minimal medium

recombinant LGK is overexpressed and its molecular mass is similar to theoretic calculation $48.4 \mathrm{kDa}$ (Fig. 3). Furthermore, in liquid M9 MM with $1.0 \% \mathrm{LG}$ as a sole carbon source, recombinant $\mathrm{pET}$-lgk would grow and reach stationary phase about $15 \mathrm{~h}$ under $0.1 \mathrm{mM}$ IPTG. As shown in Fig. 4, pET-lgk can grow in M9 MM plate, while pTanmK and pET-21a cannot. This indicates that recombinant LGK has activity for LG and AnmK has not, although they have similar catalytic mechanism.

\section{Properties of recombinant LGK}

The optimum temperature and $\mathrm{pH}$ were $30^{\circ} \mathrm{C}$ and $\mathrm{pH} 9.0$, respectively (Fig. 5), which was consistent with natural LGK (Ning et al. 2008). Compared with natural LGK, recombinant LGK seems to be more thermostable, for recombinant LGK still had $57 \%$ relative activity after incubation at $35^{\circ} \mathrm{C}$, while natural LGK lost its activity rapidly. In addition, recombinant LGK nearly had no activity at $\mathrm{pH} 6.5$, while natural LGK had $20 \%$ relative activity. Recombinant LGK was stable between pH 7-10 as natural LGK.

Recombinant LGK had a higher apparent $K_{\mathrm{m}}$ value for LG $(105.3 \pm 12.5 \mathrm{mM})$ than natural LGK $(68.6 \mathrm{mM})$, whereas the $K_{\mathrm{m}}$ value for ATP was similar $(0.20 \pm 0.02$ $\mathrm{mM}) .{ }^{1}$ Presumably, natural LGK would be an oligomer containing binding partner that is important for the activity of LGK.

It was previously shown that LGK had no activity for various analogs of LG including D-glucosamine (GlcN), $N$ acetyl-D-glucosamine (GlcNAc), 1,6-anhydro- $\beta$-D-galactopyranose, 1,6-anhydro- $\beta$-D-mannopyranose, 1,6-anhydromaltose and 2,7-anhydro- $\beta$-D-altro-sedoheptulose, and these compounds had no inhibitions to the enzymatic activity of LGK (Kitamura and Yasui 1991). We assumed that anhGlcN or anhGlcNAc might be the substrate of

\footnotetext{
1 The $K_{\mathrm{m}}$ value of natural LGK for ATP was $0.68 \mathrm{mM}$ by Ning et al. (2008). We measured it again and affirmed that it was $0.20 \mathrm{mM}$.
}

LGK, for they are transitional structural analogs between LG and anhMurNAc. However, in vitro, anhGlcN or anhGlcNAc had no inhibition to the enzymatic activity of LGK. In vivo, L. starkeyi does neither utilize them, nor GlcN and GlcNAc, which suggests that L. starkeyi would have no metabolism pathways for them in the cell or they could not enter the cell. In addition, Table 1 indicates that LGK has low activity for them and AnmK has faint activity for anhGlcNAc. It's reasonably deduced that LGK should be more compatible with LG than with anhGlcN or anhGlcNAc and shows substrate specificity for LG, while AnmK shows stricter substrate specificity.

In contrast to the lgk genes previously isolated (Zhang and Zhang, 2002; Xie et al. 2005), in this study, the deduced amino sequence of $l g k$ gene is homologous to that of bacteria's AnmK, whose catalytic mechanism is similar to that of LGK, in addition, the crude extract had high activity and recombinant $E$. coli could grow in M9 MM with LG as a sole carbon source. This implies that they are different $l g k$ genes, and the $l g k$ gene isolated in this study is a novel and more useful gene for the bioconversion of LG.

Owing to LGK's low affinity for LG, direct bioconversion of LG is apparently bottlenecked. Thus, it is necessary to reconstruct LGK to enhance the affinity for LG. Recently, in the AnmK family, the 3D structure of AnmK from Shewanella oneidensis MR-1 was resolved with the method of X-ray Diffraction (Protein Data Bank accession no. 3cqy). It is promising to elucidate the detailed catalytic mechanism of LGK by virtue of homolog-modeling and to devise a high affinity enzyme for LG, and to construct efficient genetic microbial strains to convert LG to useful products such as ethanol. These are in progress.

Acknowledgments This work was supported by the National Science Foundation of China (No. 20507021 and No. 40603024) and in part by the President Fund of GUCAS (No. M3018). We are grateful to Professor Dr. James T. Park (Tufts University, USA) for his generous present: E. coli BL21 (DE3)/pTanmK, and helpful private communications. 


\section{References}

Adams A, Gottschling DE, Kaiser CA, Stearns T (2001) Methods in yeast genetics: a cold spring harbour laboratory course manual. Cold Spring Harbor Laboratory Press, NY

Brown RC, Radlein D, Piskorz J (2001) Pretreatment processes to increase pyrolytic yield of levoglucosan from herbaceous feedstocks. In: Bosell JJ (ed) Chemicals and materials from renewable resources. American Chemical Society, Washington, DC, pp 123-134

Farrell AE, Plevin RJ, Turner BT, Jones AD, O'Hare M, Kammen DM (2006) Ethanol can contribute to energy and environmental goals. Science 311:506-508

Gao S, Hegg DA, Hobbs PV, Kirchstetter TW, Magi BI, Sadilek M (2003) Water-soluble organic components in aerosols associated with savanna fires in southern Africa: identification, evolution, and distribution. J Geophys Res 108:8491-8506

Kitamura Y, Yasui T (1991) Purification and some properties of levoglucosan (1,6-anhydro- $\beta$-D-glucopyranose) kinase from the yeast Sporobolomyces salmonicolor. Agric Biol Chem 55:523-529

Kitamura Y, Abe Y, Yasui T (1991) Metabolism of levoglucosan (1,6-anhydro- $\beta$-D-glucopyranose) in microorganisms. Agric Biol Chem 55:515-521

Nakagawa M, Sakai Y, Yasui T (1984) Itaconic acid fermentation of levoglucosan. J Ferment Technol 62:201-203

Nakahara K, Kitamura Y, Yamagishi Y, Shoun H, Yasui T (1994) Levoglucosan dehydrogenase involved in the assimination of levoglucosan in Arthrobacter sp. I-552. Biosci Biotechnol Biochem 58:2193-2196

Ning J, Yu Z, Xie H, Zhang H, Zhang G, Bai Z, Yang S, Jiang Y (2008) Purification and characterization of levoglucosan kinase from Lipomyces starkeyi YZ-215. World J Microbiol Biotechnol $24: 15-22$

Prosen EM, Radlein D, Piskorz J, Scott DS, Legge RL (1993) Microbial utilization of levoglucosan in wood pyrolysate as a carbon and energy source. Biotechnol Bioeng 42:538-541

Sambrook J, Russell DW (2001) Molecular cloning: a laboratory manual, 3rd edn. Cold Spring Harbor Laboratory Press, NY
Schauer JJ, Kleeman MJ, Cass GR, Simoneit BRT (2001) Measurement of emissions from air pollution sources. 3. C1-C29 organic compounds from fireplace combustion of wood. Environ Sci Technol 35:1716-1728

Thompson JD, Gibson TJ, Plewniak F, Jeanmougin F, Higgins DG (1997) The clustalX windows interface: flexible strategies for multiple sequence alignment aided by quality analysis tools. Nucleic Acids Res 25:4876-4882

Uehara T, Suefuji K, Valbuena N, Meehan B, Donegan M, Park JT (2005) Recycling of the anhydro- $N$-acetylmuramic acid derived from cell wall murein involves a two-step conversion to $\mathrm{N}$-acetylglucosamine-phosphate. J Bacteriol 187:3643-3649

Waltz E (2008) Cellulosic ethanol booms despite unproven business models. Nat Biotechnol 26:8-9

Xie H, Zhuang X, Zhang H, Bai Z, Qi H (2005) Screening and identification of the levoglucosan kinase gene $(l g k)$ from Aspergillus niger by LC-ESI-MS/MS and RT-PCR. FEMS Microbiol Lett 251:313-319

Xie H, Zhuang X, Bai Z, Qi H, Zhang H (2006) Isolation of levoglucosan-assimilating microorganisms from soil and an investigation of their levoglucosan kinases. World J Microbiol Biotechnol 22:887-892

Yu Z (2003) Fermentation of cellulosic pyrolysis products to ethanol and its construction of cell engineering strain. Dissertation, Graduate University of the Chinese Academy of Sciences

Yu Z, Zhang H (2003) Ethanol fermentation of acid-hydrolyzed cellulosic pyrolysate with Saccharomyces cerevisiae. Bioresour Technol 90:95-100

Zhuang X, Zhang H (2002) Identification, characterization of levoglucosan kinase, and cloning and expression of levoglucosan kinase cDNA from Aspergillus niger CBX-209 in Escherichia coli. Protein Express Purific 26:71-81

Zhuang XL, Zhang HX, Tang JJ (2001) Levoglucosan kinase involved in citric acid fermentation by Aspergillus niger CBX209 using levoglucosan as sole carbon and energy source. Biomass Bioenergy 21:53-60 